\title{
Recomendaciones para el consumo de endulzantes no calóricos. ¿Basadas en normas regulatorias o en evidencia científica?
}

\author{
Recommendations for the consumption of non-caloric sweeteners. Based on regulatory \\ standards or scientific evidence?
}

Diana Hidalgo-Silva ${ }^{a}$, Marcos Galván ${ }^{b}$, Marco González-Unzaga ${ }^{c}$ y Diana P. Olivo-Ramírez ${ }^{d}$ Abstract:

Given the increasing global prevalence of overweight and obesity, the total or partial replacement of sugars in the food industry by low-calorie sweeteners had as its main motivation the decrease in the energy intake of sugary foods and beverages, in order to reduce energy intake and contribute to weight loss. Current evidence suggests that consumption of non-caloric sweeteners may have a beneficial effect on weight control, however experts admit that further research is necessary to ensure the existence of a positive effect on human health. This work provides the reader, especially nutritionists and doctors, with updates to improve selection criteria and / or recommendations for the use of non-caloric sweeteners.

\section{Keywords:}

Non-caloric sweeteners, non-nutritive sweeteners, weight control, energy intake

\section{Resumen:}

Ante las crecientes prevalencias mundiales de sobrepeso y obesidad, el reemplazo total o parcial de los azúcares en la industria alimentaria por endulzantes bajos en calorías tuvo como principal motivación la disminución del aporte energético de alimentos y bebidas azucaradas, con el fin de reducir la ingesta energética y contribuir a la pérdida de peso. La evidencia actual sugiere que el consumo de endulzantes no calóricos puede tener un efecto benéfico en el control de peso, no obstante expertos reconocen que es necesaria mayor investigación para asegurar la existencia de un efecto positivo en la salud humana. Este trabajo brinda al lector, en especial nutricionistas y médicos, actualizaciones para la mejora de criterios de selección y/o recomendaciones para el uso de endulzantes no calóricos.

\section{Palabras Clave:}

Endulzantes no calóricos, control de peso, ingesta energética.

\section{Introducción}

En las últimas dos décadas, en México y en el mundo, ha ido aumentando paulatinamente la tendencia a consumir alimentos light que aportan menos calorías, los cuales son percibidos por la población como "más saludables". De acuerdo con la empresa Nielsen la cual estudia las tendencias y hábitos de consumo en más de cien países (1) en México al menos el $10 \%$ de los hogares se compraron productos alimenticios, denominados wellness (categorizados como light, saludables, orgánicos y funcionales), para buscar o mantener un estilo de vida sano. Estas compras incrementaron aproximadamente en un $45 \%$ el gasto anual en alimentos, en estos hogares (2), significando una fuerte inversión que puede no corresponder con los beneficios en salud obtenidos.

Es posible que el interés por el consumo de productos wellness o más saludables esté motivado en la población por la alarmante prevalencia de sobrepeso y obesidad, diabetes, hipertensión, hipertrigliceridemia e

\footnotetext{
a Universidad Autónoma del Estado de Hidalgo, https://orcid.org/0000-0001-9054-6872, Email: dianghidalgo@ gmail.com

b Universidad Autónoma del Estado de Hidalgo, https://orcid.org/0000-0002-3254-4470, Email: marcos_galvan3112@uaeh.edu.mx c Universidad Autónoma del Estado de Hidalgo, https://orcid.org/0000-0002-4355-8456, Email: marcounzaga@gmail.com

d Autor de Correspondencia, Universidad Autónoma del Estado de Hidalgo, https://orcid.org/0000-0001-5184-8202, Email: diana_olivo@uaeh.edu.mx
} 
hipercolesterolemia presente en los mexicanos mayores a 20 años (3) y además por la creciente tendencia a consumir productos que no dañen el ambiente.

Por su parte, la industria alimentaria optó por disminuir el aporte calórico final de sus productos con el empleo de endulzantes no calóricos (ENC) en reemplazo o disminución de azúcares. Lo anterior debido a los efectos negativos en la salud que se han asociado con el consumo de alimentos industrializados, entre ellos la obesidad, y para evitar la imposición de impuestos a ciertos productos por su alto aporte de calorías. Con la introducción de los ENC a esta industria, ha surgido un debate científico acerca del impacto, positivo o negativo, que estos aditivos pueden tener sobre la salud humana.

\section{Uso de endulzantes no calóricos}

Los endulzantes son sustancias que se utilizan en los productos alimenticios para conferirles un sabor dulce. Se clasifican de acuerdo con su origen en naturales o artificiales y, de acuerdo con su aporte nutritivo en calóricos o no calóricos. Adicionalmente, cabe mencionar que los edulcorantes no calóricos se caracterizan por poseer un alto poder de dulzor, cientos de veces más alto, en comparación con la sacarosa, edulcorante natural y calórico comúnmente conocido como azúcar de mesa.

Como ya fue mencionado, la industria, como estrategia para disminuir el aporte energético de los azúcares (endulzantes naturales, calóricos) en los alimentos industrializados, emplea ENC como aditivos alimentarios en sustitución o reemplazo parcial o total de los endulzantes calóricos. De hecho, existen innumerables productos alimenticios que han sido reformulados, de tal manera que una vasta mayoría de productos, en especial las bebidas, cuentan con su versión comercial "reducida en calorías" que puede tener uno o varios ENC. Asimismo, existen presentaciones de estos compuestos "en sobres" que se ofrecen como sustitutos de azúcar para su uso cotidiano.

En lo que respecta a México, la Comisión Federal para la Protección contra Riesgos Sanitarios (COFEPRIS) regula mediante la norma NOM-218- SSA1-2011 el empleo de Ios ENC como aditivos alimentarios, siendo los permitidos: aspartame, acesulfame potásico, sucralosa, sacarina, glucósidos de esteviol, ciclamato, alitame, neotame, advantame y alulosa (4). Asimismo, los ENC se consideran dentro de la NOM-015-SSA2-2018, la cual trata sobre la prevención, tratamiento y control de la diabetes mellitus, denominándolos "edulcorantes no nutritivos" y definiéndolos como: aditivos que son endulzantes potentes, con aporte energético es mínimo y que no afectan los niveles de insulina o glucosa sérica (5) Cabe mencionar que la norma no detalla indicaciones o contraindicaciones para el uso de ENC por personas con obesidad, hipertensión o para su uso por mujeres gestantes o en periodos de lactancia o bien indicaciones por grupo de edad.

Aunque la seguridad y aprobación para el consumo de los diversos ENC está basada en una amplia investigación toxicológica, el hecho de que los ENC no son moléculas inertes, sino que son sustancias que se metabolizan o bien que tienen interacciones con receptores del sabor dulce localizados oralmente y en tejidos extraorales, ha llevado a la búsqueda de alteraciones fisiológicas provocadas por estos compuestos, en modelos in vivo e in vitro $(6)(7)(8)(9)(10)(11)$. Los resultados de estos modelos, iniciaron el debate sobre la verdadera seguridad de los ENC, no obstante, los escasos estudios clínicos y las fallas metodológicas en estos no han aportado evidencia sustancial para negar que los ENC son seguros para su uso humano.

\section{Endulzantes no calóricos: ¿el consenso de los expertos es definitivo?}

A finales de 2018 en Londres, se llevó a cabo una reunión de expertos para analizar la evidencia clínica disponible actualmente sobre endulzantes no calóricos y la seguridad en su consumo, así como su efecto sobre el control de peso corporal y el control de la glicemia. El consenso de expertos concluyó que es posible que los ENC sean útiles para mejorar el control de peso y de la diabetes, así como el cumplimiento de la dieta. No obstante, también reconoce que, aunque existe evidencia respecto a la seguridad del uso humano de ENC, son necesarios más estudios para dilucidar por completo los beneficios y de los riesgos para la salud, así como de las pautas para su consumo (12).

Esta última sentencia, invita a la investigación para esclarecer el balance costo/beneficio a la salud que involucra el empleo de ENC (ya que faltan estudios sobre su empleo a largo plazo). Además, advierte de la necesidad de establecer las pautas para su consumo en grupos de población que enfrentan eventos fisiológicos y metabólicos, distintos a las individuos en los que se ha realizado las intervenciones clínicas, tales como las mujeres gestantes y en periodo de lactancia, lactantes, infantes, personas con sobrepeso u obesidad, diabetes o hipertensión, y/o con alguna otra complicación metabólica, en las cuales es más difícil dilucidar el verdadero efecto que causan los ENC, pues siempre existe el factor de causalidad inversa.

En relación con lo que el consenso de expertos concluye, en cuanto a la posible contribución del empleo de ENC para mejorar el control de peso (12), se ha comprobado que, en cantidades pequeñas, los ENC logran un bajo efecto benéfico para el control de peso en individuos adultos y sanos: a menores dosis, menor ganancia de peso en contraste a altas dosis que muestran mayor ganancia de peso (13). Por otra parte, según Cavagnari 
(2019) el consumo de ENC en la mujer gestante, no representa un efecto benéfico para el control de peso, sino por el contrario, su consumo se ha asociado con aumento de peso (14). De igual manera, el consumo de ENC durante el embarazo, presenta una asociación positiva con sobrepeso y la ocurrencia de obesidad en la descendencia a los 7 años de edad (15). En otras palabras, lo anterior indica que la capacidad para fungir como coadyuvantes en la reducción-control de peso es escasa, y no aplica para el grupo de las mujeres embarazadas, en quienes es posible que las perturbaciones metabólicas maternas que su empleo puedan provocarles durante la gestación, predispongan a los vástagos al sobrepeso y desórdenes metabólicos más tarde en la vida.

Por otra parte, con base en evidencia clínica obtenida mediante resonancia magnética funcional, un grupo de investigación en Noruega, propuso que los ENC no estimulan como los endulzantes calóricos, en específico la glucosa, al sistema hedónico de recompensa, por lo que la dopamina no es liberada, lo que tiene como consecuencia una mayor ingesta calórica. Cuando se presenta la respuesta de saciedad en el cerebro, se observa una disminución en la demanda de oxígeno en el hipotálamo, lo cual solo se logra mediante la estimulación de las células dopaminérgicas sensibles a glucosa. En particular, la sucralosa, un ENC cuya estructura semeja a la sacarosa, incrementa la saturación de oxígeno en el hipotálamo, lo cual representa una respuesta de hambre y probablemente cause en el individuo un mayor consumo de otros alimentos para llegar a ese estado de saciedad que demanda el cuerpo (16).

Apenas en marzo de 2020, investigadores norteamericanos en colaboración con este mismo grupo noruego, reportaron que el consumo de sucralosa, en combinación con carbohidratos, afecta la sensibilidad a la insulina, afección metabólica que fue asociada a una disminución en la respuesta neuronal al azúcar. La afección a la sensibilidad a la insulina no fue observada cuando se suministraron por separado la sucralosa y los carbohidratos (17). Este y otros hallazgos, como la modificación al microbioma intestinal (18), la diferenciación de pre-adipocitos a adipocitos (19) y la presencia de estas sustancias en leche materna (20) fortalecen el planteamiento de la necesidad de seguir investigando los efectos fisiológicos de los ENC, a quienes en el pasado se les consideraba como moléculas fisiológicamente inertes.

\section{Conclusiones}

La recomendación o contraindicación del consumo de ENC debe justificarse a la luz de la investigación clínica que aporte evidencia sustancial para subsanar las interrogantes que surgen acerca su efecto en la fisiología y patologías humanas en diferentes etapas de la vida. Es necesario que los organismos gubernamentales encargados de normar el consumo de ENC cuenten con información basada en evidencia científica para recomendar o contraindicar este consumo. Desafortunadamente, generar esta evidencia tomará aún más años de investigación. Mientras tanto, es crucial que los profesionales de la salud (en especial nutricionistas y médicos), manteniendo siempre el enfoque de mejorar la salud de sus pacientes, antes de indicar el consumo de productos con ENC, avancen a la nutrición personalizada, y emitan sus recomendaciones de manera actualizada e individualizada (considerando y respetando las prácticas culturales), no necesariamente estando sujetos solo a lo que dictan las normas alimentarias.

\section{Referencias}

1. Nielsen.com.mx [Internet]. [cited 2020 Apr 15]. Available from: https://www.nielsen.com/mx/es/about-us/

2. Forbes México [Internet]. 2019. Available from: https://www.forbes.com.mx/mexicanos-gasto-saludable/

3. Secretaría de Salud, Instituto Nacional de Salud Pública (INSP) IN de E y G. Encuesta Nacional de Salud y Nutrición- 2018. Presentacipón de resultados. Ciudad de México; 2019.

4. Secretaría de Gobernación DO de la F. NOM-218-SSA1-2011. NOM218-SSA1-2011. 2011.

5. Gobernación S de. PROY-NOM-015-SSA2-2018. Diario Oficial de la Federación. 2018.

6. Rother KI, Conway EM, Sylvetsky AC. How Non-nutritive Sweeteners Influence Hormones and Health. Vol. 29, Trends in Endocrinology and Metabolism. Elsevier Inc.; 2018. p. 455-67.

7. Rother KI, Sylvetsky AC, Schiffman SS. Non-nutritive sweeteners in breast milk: perspective on potential implications of recent findings. Vol. 89, Archives of Toxicology. Springer Verlag; 2015. p. 2169-71.

8. Toews I, Lohner S, Küllenberg De Gaudry D, Sommer H, Meerpohl JJ. Association between intake of non-sugar sweeteners and health outcomes: Systematic review and meta-analyses of randomised and non-randomised controlled trials and observational studies. BMJ. $2019 ; 364$.

9. Archibald AJ, Dolinsky VW, Azad MB. Early-life exposure to nonnutritive sweeteners and the developmental origins of childhood obesity: Global evidence from human and rodent studies. Nutrients. 2018;10(2).

10. Sylvetsky AC, Figueroa J, Rother KI, Goran MI, Welsh JA. Nutritional Epidemiology and Public Health Trends in Low-Calorie Sweetener Consumption Among Pregnant Women in the United States. 2019.

11. Myers EA, Duncan SE, Wang A, Hedrick VE. Identifying an appropriate carrier for nonnutritive sweeteners in metabolic and controlled feeding investigations via sensory evaluation. J Sens Stud. 2019;34(2):1-8

12. Ashwell M, Gibson S, Bellisle F, Buttriss J, Drewnowski A, Fantino M, et al. Expert consensus on low-calorie sweeteners: Facts, research gaps and suggested actions. Nutr Res Rev. 2019;

13. Shannon M, Rehfeld A, Frizzell C, Livingstone C, McGonagle C, Skakkebaek NE, et al. In vitro bioassay investigations of the endocrine disrupting potential of steviol glycosides and their metabolite steviol, components of the natural sweetener Stevia. Mol Cell Endocrinol. 2016 May 15;427:65-72. 
14. Cavagnari BM. [Non-caloric sweeteners in pregnancy and lactation] Rev Esp Salud Publica. 2019;93.

15. Zhu Y, Olsen SF, Mendola P, Halldorsson TI, Rawal S, Hinkle SN, et al. Maternal consumption of artificially sweetened beverages during pregnancy, and offspring growth through 7 years of age: A prospective cohort study. Int J Epidemiol. 2017;46(5):1499-508.

16. Van Opstal AM, Hafkemeijer A, van den Berg-Huysmans AA, Hoeksma M, Mulder TPJ, Pijl H, et al. Brain activity and connectivity changes in response to nutritive natural sugars, non-nutritive natural sugar replacements and artificial sweeteners. Nutr Neurosci. 2019;0(0):1-11.

17. Dalenberg JR, Patel BP, Denis R, Veldhuizen MG, Nakamura Y, Vinke $\mathrm{PC}$, et al. Short-Term Consumption of Sucralose with, but Not without, Carbohydrate Impairs Neural and Metabolic Sensitivity to Sugar in Humans. Cell Metab. 2020;31(3):493-502.e7

18. Bian X, Chi L, Gao B, Tu P, Ru H, Lu K. The artificial sweetener acesulfame potassium affects the gut microbiome and body weight gain in CD-1 mice. PLoS One. 2017 Jun 1;12(6).

19. Simon BR, Parlee SD, Learman BS, Mori H, Scheller EL, Cawthorn WP, et al. Artificial sweeteners stimulate adipogenesis and suppress lipolysis independently of sweet taste receptors. J Biol Chem. 2013;288(45):32475-89.

20. Rother KI, Sylvetsky AC, Walter PJ, Garraffo HM, Fields DA Pharmacokinetics of Sucralose and Acesulfame-Potassium in Breast Milk Following Ingestion of Diet Soda. J Pediatr Gastroenterol Nutr. 2018 Mar 1;66(3):466-70. 